\title{
Editorial
}

\section{Unmet Needs in the Field of Neuroendocrine Neoplasms of the Gastrointestinal Tract, Pancreas, and Respiratory System: Reports by the ENETS Group}

\author{
Wouter W. de Herder ${ }^{a} \quad J a u m e$ Capdevila ${ }^{b}$ \\ a Department of Internal Medicine, Erasmus MC, ENETS Center of Excellence, Erasmus MC Cancer Center, Rotterdam, \\ The Netherlands; 'ball d'Hebron Institute of Oncology (VHIO), Vall d'Hebron University Hospital, Barcelona, Spain
}

On the 10th and 11th of November 2016, 54 international experts in the field of neuroendocrine neoplasms (NEN) of the gastrointestinal tract, pancreas, and respiratory system met in Munich, Germany, to discuss and determine unmet needs in this field. The participating experts were Advisory Board members and invited guests of the European Neuroendocrine Tumor Society (ENETS). The Board members were medical specialists with different clinical or preclinical backgrounds, basic scientists, specialized nurses, and patient representatives.

According to the Oxford dictionary, "unmet" refers to something (here, a need) which has not been achieved or fulfilled. The NEN expert subgroups succeeded in producing 6 reports on what they identified as the most relevant unmet needs in NEN. They identified several limitations of the current tumor classification, grading, and staging systems for almost all NEN subtypes.

Apart from the historical separation regarding origin into foregut, midgut, and hindgut, other subentities have now been recognized, each requiring a more tailored approach. There is still a general lack of predictive and prognostic markers, in samples of both tumor tissue and surrogate tissues such as the blood. Tumor visualization making use of radiology and nuclear medicine has seen impressive advances in recent years. However, with regard to determining tumor (metastases) responses, there

\section{KARGER}

E-Mail karger@karger.com www.karger.com/nen

\section{(C) 2018 The Author(s)}

Published by S. Karger AG, Basel

This article is licensed under the Creative Comm This article is licensed under the Creative Commons AttributionNonCommercial-NoDerivatives 4.0 International License (CC BY NC-ND) (http://www.karger.com/Services/OpenAccessLicense). Usage and distribution for commercial purposes as well as any distribution of modified material requires written permission. is still debate as to how best to achieve this with different imaging modalities.

In spite of more treatment options (locoregional and systemic) being available for metastatic NENs, help is needed to select appropriate treatment strategies (sequencing) and there is also a need for more effective treatments. For some tumor entities, watchful waiting might be considered (e.g., in the case of small pancreatic NENs) but, again, the lack of reliable markers makes it difficult to decide which tumors should undergo resection and which should be monitored only. There are currently no registered or approved adjuvant treatment options for the period after curative resection of some of the isolated or locally advanced NENs (e.g., of the pancreas, appendix, and rectum). It is also unclear which patients might benefit from adjuvant treatment. The follow-up of patients with NENs remains a poorly studied area and is based on loose empirical international guidelines or institutional opinion.

All of these unmet needs in the field of NENs exponentially increase the complexity of conducting well-designed clinical and translational studies to resolve these issues, with multidisciplinary and international efforts such as the current one led by ENETS being the only way to move forward. We invite all Readers of Neuroendocrinology interested in the field of NENs to study the series of 6 articles reporting on unmet needs in the field of NENs [1-6]. 


\section{References}

1 Baudin E, Hayes A, Scoazec JY, Filosso PL, Lim E, Kaltsas G, et al; ENETS 2016 Munich Advisory Board Participants. Unmet medical needs in pulmonary neuroendocrine (carcinoid) neoplasms. Neuroendocrinology. 2019; 108:7-17.

2 Jensen RT, Bodei L, Capdevila J, Couvelard A, Falconi M, Grozinsky-Glasberg S, et al; ENETS 2016 Munich Advisory Board Participants. Unmet Needs in Functional and Nonfunctional pancreatic neuroendocrine neoplasms(PanNENs). Neuroendocrinology. 2019;108:26-36.
3 Capdevila J, Bodei L, Davies P, Gorbounova V, Jensen RT, Knigge U, et al; ENETS 2016 Munich Advisory Board Participants. Unmet medical needs in metastatic lung and digestive neuroendocrine neoplasms. Neuroendocrinology. 2019;108:18-25.

4 Toumpanakis C, Fazio N, Tiensuu Janson E, Hörsch D, Pascher A, Reed N, et al; ENETS 2016 Munich Advisory Board Participants. Unmet Needs in Appendiceal Neuroendocrine Neoplasms. Neuroendocrinology. 2019;108:37-44.
5 Ramage JK, Valle J, Nieveen van Dijkum EJ, Sundin A, Pascher A, Couvelard A, et al; ENETS 2016 Munich Advisory Board Participants. Colorectal Neuroendocrine Neoplasms - areas of unmet need. Neuroendocrinology. 2019;108:45-53.

6 Sorbye H, Baudin E, Borbath I, Caplin M, Chen J, Cwikla J, et al. ENETS 2016 Munich Advisory Board Participants. Unmet needs in high-grade gastroenteropancreatic neuroendocrine neoplasms (WHO G3). Neuroendocrinology. 2019;108:54-62. 